\title{
Activation of an AMP-activated protein kinase is involved in post-diapause development of Artemia franciscana encysted embryos \\ Xiao-Jing Zhu ${ }^{1,2}$, Jie-Qiong Dai1,2, Xin Tan³, Yang Zhao ${ }^{1,2}$ and Wei- Jun Yang*1,2
}

\begin{abstract}
Address: 'Institute of Cell Biology and Genetics, College of Life Sciences, Zijingang Campus, Zhejiang University, Hangzhou, Zhejiang 310058 , PR China, ${ }^{2}$ State Conservation Center for Gene Resources of Wildlife, Key Laboratory of Conservation Genetics and Reproductive Biology for Wild Animals of the Ministry of Education, Hangzhou, Zhejiang 310058, PR China and ${ }^{3}$ College of Life Sciences, Zijingang Campus, Zhejiang University, Hangzhou, Zhejiang 310058, PR China

Email: Xiao-Jing Zhu - xiao_jingzhu@hotmail.com; Jie-Qiong Dai - coral_dai@hotmail.com; Xin Tan - tanxin812@gmail.com; Yang Zhao - zhaoyang85211@sina.com; Wei-Jun Yang*-w_jyang@cls.zju.edu.cn

* Corresponding author
\end{abstract}

Published: 16 March 2009

BMC Developmental Biology 2009, 9:21 doi:10.1186/147I-213X-9-2I
Received: 29 July 2008

Accepted: 16 March 2009

This article is available from: http://www.biomedcentral.com/I47I-2I3X/9/2I

(c) 2009 Zhu et al; licensee BioMed Central Ltd.

This is an Open Access article distributed under the terms of the Creative Commons Attribution License (http://creativecommons.org/licenses/by/2.0), which permits unrestricted use, distribution, and reproduction in any medium, provided the original work is properly cited.

\begin{abstract}
Background: Cysts of Artemia can remain in a dormant state for long periods with a very low metabolic rate, and only resume their development with the approach of favorable conditions. The post-diapause development is a very complicated process involving a variety of metabolic and biochemical events. However, the intrinsic mechanisms that regulate this process are unclear.

Results: Herein we report the specific activation of an AMP-activated protein kinase (AMPK) in the post-diapause developmental process of Artemia. Using a phospho-AMPK $\alpha$ antibody, AMPK was shown to be phosphorylated in the post-diapause developmental process. Results of kinase assay analysis showed that this phosphorylation is essential for AMPK activation. Using wholemount immunohistochemistry, phosphorylated AMPK was shown to be predominantly located in the ectoderm of the early developed embryos in a ring shape; however, the location and shape of the activation region changed as development proceeded. Additionally, Western blotting analysis on different portions of the cyst extracts showed that phosphorylated AMPK $\alpha$ localized to the nuclei and this location was not affected by intracellular $\mathrm{pH}$. Confocal microscopy analysis of immunofluorescent stained cyst nuclei further showed that AMPK $\alpha$ localized to the nuclei when activated. Moreover, cellular AMP, ADP, and ATP levels in developing cysts were determined by HPLC, and the results showed that the activation of Artemia AMPK may not be associated with cellular AMP:ATP ratios, suggesting other pathways for regulation of Artemia AMPK activity.
\end{abstract}

Conclusion: Together, we report evidence demonstrating the activation of AMPK in Artemia developing cysts and present an argument for its role in the development-related gene expression and energy control in certain cells during post-diapause development of Artemia. 


\section{Background}

Artemia is a species of primitive crustaceans capable of producing diapause encysted embryos (cysts) to survive adverse conditions. The cyst, composed of about 4000 cells and developmentally arrested at the gastrula stage, is remarkably resistant to physiologic stressors [1]. Diapause embryos remain in dormancy and will not resume development until they are activated by transient exposure to a specific environmental stimulus. Activated cysts require only suitable environmental conditions to resume metabolism and development, and eventually emerge as fully formed nauplii [2]. The special pattern of the cyst development mades it an ideal system for biological study.

Thus far, the sequence of events accompanying the diapause and resumption of development in Artemia has been investigated extensively. Two proteins, p26 and artemin, are present in large amounts in encysted embryos. p26 exhibits reversible nuclear-cytoplasmic translocation and plays an important role as a molecular chaperone, while artemin is a RNA-binding protein with high thermal stability and may act as a RNA chaperone [1,3-5]. Previous studies have also suggested intracellular $\mathrm{pH}(\mathrm{pHi})$ as a key cellular signal in the metabolic and developmental switching [6,7]. Interestingly, post-diapause development takes place in the absence of DNA synthesis and cell division $[8,9]$, and is known to be a very complicated process involving a variety of metabolic events. These events include the catabolism of trehalose, degradation of yolk platelets, protein synthesis, gene transcription, and other events, coupled with a large number of energy changes [10-14]. However, the intrinsic mechanisms of this complicated process remain unclear.

Adenosine monophosphate-activated protein kinase (AMPK) is a cellular energy sensor that is conserved throughout eukaryotes. AMPK also plays an important role in the control of the whole body's energy balance $[15,16]$. AMPK homologues exist as heterotrimeric complexes consisting of a catalytic $\alpha$-subunit and non-catalytic $\beta$ - and $\gamma$-subunits [16]. Activation of AMPK absolutely requires phosphorylation at a specific threonine residue (Thr-172) of the $\alpha$-subunit by upstream kinases (LKB1 or CaMKK $\beta$ ), and allosterically by increases in the AMP:ATP ratio $[17,18]$. AMPK activation may also be elicited by other cellular signals, such as glycogen [19].

The upstream kinase, LKB1, signals through AMPK to regulate multiple metabolic processes. There is also evidence that AMPK has a more complex role in the regulation of diverse cellular processes, including the cell cycle, proliferation, and others through the LKB1 $\rightarrow$ AMPK pathway [15]. The differential tissue-specific and subcellular localization of AMPK is critical in investigating its functions. In general, nuclear AMPK activation may elicit long-term changes in gene expression, whereas cytosolic AMPK may function in the modulation of more immediate metabolic and homeostatic responses [19].

The main objective of this study was to explore the role of AMPK in the Artemia life cycle. In a previous study, we have identified an Artemia AMPK gene (Afr-AMPK 1) which is differentially expressed during the developmental stages [20]. Herein we report the activation of AMPK in Artemia post-diapause developmental stages and discuss its possible roles in this process.

\section{Results \\ AMPK activation during the post-diapause developmental stages of Artemia}

In our previous study, an Artemia AMPKa gene (AfrAMPKalpha1) was isolated and the pattern of the gene expression was studied [20]. The deduced amino acid sequence near threonine (172) in the T-loop region of this protein is identical with human AMPKa. Thus, a phospho-AMPKa (Thr172) antibody designed corresponding to the residues surrounding Thr172 of human AMPKa and widely used in detecting activated AMPK in various organisms was used in the present study.

The phosphorylation of AMPK during Artemia developmental stages was analyzed by Western blotting. The results showed that AMPK is initially phosphorylated in 4$\mathrm{h}$ incubated embryos (Figure 1A). Subsequently, the phospho-AMPK level increased as the post-diapause development proceeded. No signal of phospho-AMPK was detected in the free-swimming nauplius or adults (Figure 1A). The molecular weight of the detected protein is about $60 \mathrm{kDa}$, similar to the predicted molecular weight of Afr-AMPKALPHA1 [20].

The kinase activity of AMPK was assayed with a GST-fused AMPK-specific substrate, GST-SAMS [21]. The SAMS peptide is based on the sequence surrounding serine 79 within rat acetyl-coA carboxylase and has been used most frequently for AMPK activity determination. Purified GSTSAMS was incubated with PEG extracts from embryos at different developmental stages, and the phosphorylated GST-SAMS was detected by Western blotting. As shown in Figure 1B, no signal of phosphorylated GST-SAMS was detected in 0-h incubated embryos in which phosphoAMPK was undetectable. Furthermore, the level of phospho-GST-SAMS increased as the level of phospho-AMPK increased (Figure 1B, 6-10 h). These results suggest that the activity of AMPK was elevated when the level of phosphorylated AMPK increased (Figure 1B), indicating that Artemia AMPK, like AMPK in other organisms, is activated by phosphorylation. 
A

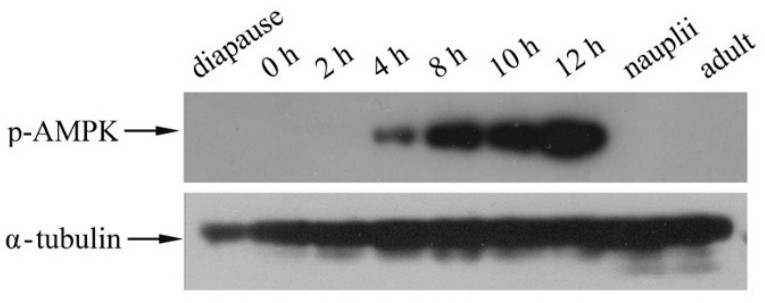

B

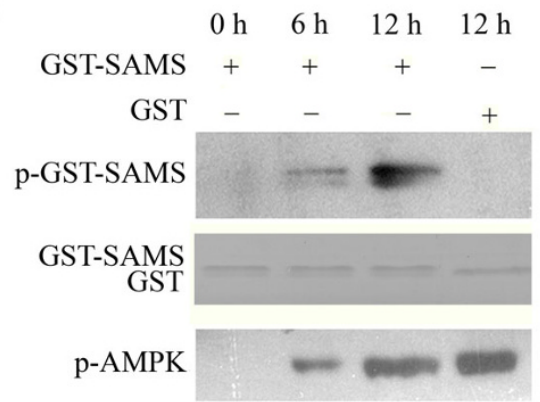

Figure 1

Activation of AMPK during developmental stages of Artemia. A. phosphorylated AMPK was detected using phospho-AMPK (Thr I72) antibody by immunoblotting extracts from diapause embryos, developing post-diapause embryos $(0-12 \mathrm{~h})$, nauplii, and adults. $\alpha$-Tubulin was used as a loading control. B. In vitro kinase assay to test AMPK activity from PEG-precipitated proteins. GST-SAMS or GST from the reaction buffer were separated by 15\% SDS-PAGE and phosphorylated proteins were detected by Western blotting using Biotinylated Phos-tag ${ }^{\mathrm{TM}}$. Coomassie staining of GST proteins as a loading control is shown in the lower panel. The presence of phosphorylated AMPK in the precipitates was determined by Western blotting with phospho-AMPK antibody. P-AMPK, phosphorylated AMPK; p-GST-SAMS, phosphorylated GST-SAMS.

Using whole-mount immunohistochemistry, we have examined the distribution of activated AMPK in developing embryos (Figure 2). The results also illustrate that AMPK is activated in 4-h embryos and embryos at later stages. Activated AMPK was predominantly located in the ectoderm region of embryos at early stages in a ring shape (Figures 2b-d). As development proceeded, the activated loop enlarged (Figure 2e), and in late embryos that have a pear shape, AMPK was activated in the head region (Figure 2f).

\section{Nuclear translocation of AMPK}

The subcellular location of activated AMPK is closely related to its function. Thus, we used a cell fraction system to separate pellet and supernatant proteins of embryos [22]. The pellet fraction loaded on SDS-PAGE contains nuclei and yolk platelets, while the supernatant contains cellular proteins. As shown in Figure 3A, activated AMPK was detectable on Western blots of pellet extracts, but not

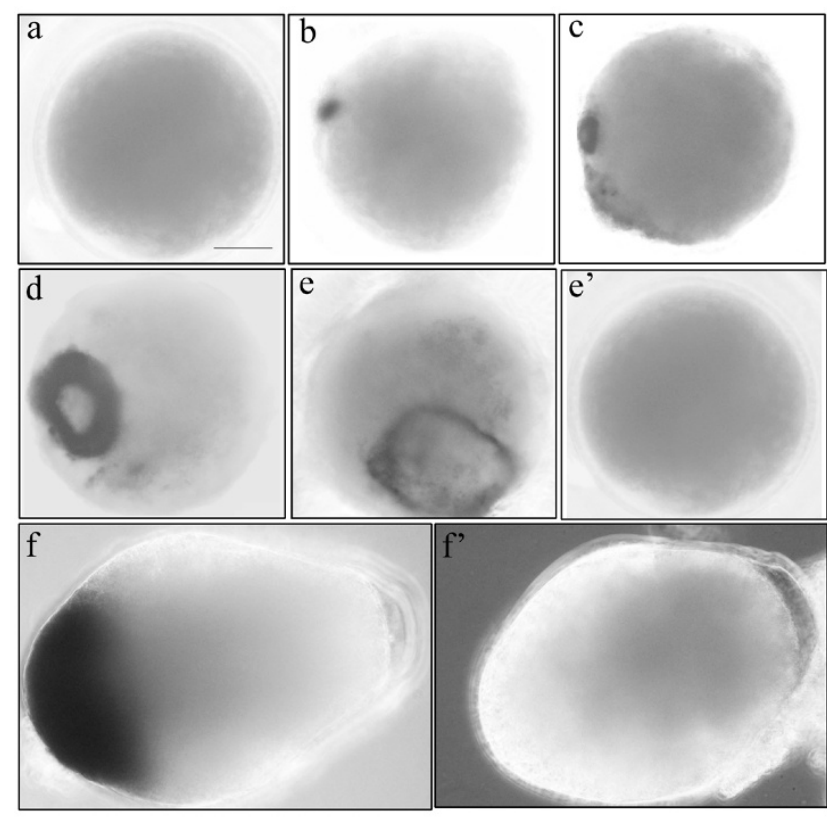

Figure 2

Location of activated AMPK in developing cysts by whole mount immunohistochemistry. Decapsulated embryos developing for $2,4,6,8,10$, and $12 \mathrm{~h}$ were fixed in $4 \%$ paraformaldehyde, permeated by $0.1 \%$ Triton X-100, incubated with phospho-AMPK (Thr I72) antibody $(a-f)$, and subsequently detected with the AP-conjugated secondary antibody by NBT/BCIP staining. $e^{\prime}$ and $f^{\prime}$ represent control embryos that were not incubated with the primary antibody. Embryos are shown at the same magnification.

in supernatant extracts. Immunofluorescent staining of nuclei and confocal microscopy further confirmed that activated AMPK was localized in the nucleus (Figure 3B; Additional file 1).

\section{Activation of AMPK is not associated with the cellular AMP:ATP ratio}

Cellular ATP, ADP, and AMP were separated by HPLC analysis in embryos at different developmental stages (Figure 4A). Concentrations of cellular adenylates were calculated by the external standard method (Additional file 2). The results showed that diapause embryos contained large amounts of AMP, while ATP and ADP were merely detectable (Figure 4A, a; Figure 4B, dp). In contrast, ATP remained at a high level in embryos during development (Figure 4B, 0-12 h). The level of ATP increased from initiation of the development until it reached its maximal value at the 8 th hour of development (Figure 4B, ATP, 0-8 h). The ATP concentration decreased in the next 4 hours, but still remained higher than at the initiation of development (Figure 4B, ATP, 8-12 h). During development, the AMP:ATP ratios were between 0.1 and 0.3 (Figure 4C). Interestingly, AMPK was not acti- 
A

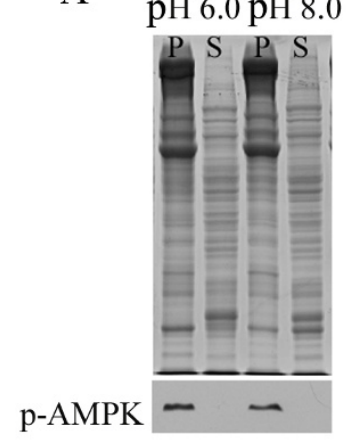

B

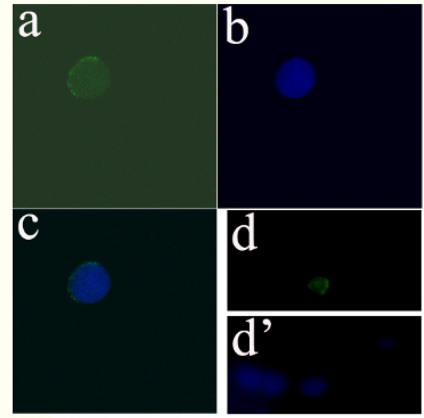

\section{Figure 3}

Subcellular location of activated AMPK. A. Supernatant $(S)$ and pellet $(P)$ fractions were prepared using buffer $\mathrm{K}(\mathrm{pH}$ 6.0 or 8.0) from 4-h incubated embryos and separated by $10 \%$ SDS-PAGE. Proteins were also transferred to PVDF membranes and detected using phospho-AMPK (Thr I72) antibody. The detected AMPK is indicated by an arrow. B. Confocal microscopy confirms that AMPK localizes in the nuclei when activated. Nuclei were double-stained using DAPI (a) and FITC-conjugated goat anti-rabbit IgG (b) after incubation with phospho-AMPK (Thr I72) antibody. c, merged image of a and $b$. $d, d$ low magnification image of a field of nuclei double-stained with FITC-conjugated goat antirabbit $\lg G(d)$ and DAPI (d').

vated in the first 4 hours in which the AMP:ATP ratio was higher than the later stages.

\section{Discussion}

AMPK is a family of serine/threonine protein kinases playing a central role in energy control, at both the cellular level and the organism as a whole [23]. Genes encoding the subunits of the AMPK complex have been identified in all eukaryotes in whom genomes have been sequenced, covering all taxa from yeast to mammals [16]. Herein we showed that Artemia AMPK is specifically activated during the post-diapause developmental stages, and that the spatial and subcellular location of activated AMPK provides valuable information for further investigation of its functions.

Post-diapause development of Artemia is a very complicated process involving a large number of internal events, including RNA and protein synthesis restoration, cellular differentiation, and associated morphologic changes $[11,12,16,24,25]$. All these biochemical reactions occur together with large energy fluctuations. Our Western blotting results showed that AMPK $\alpha$ is specifically activated during this process, but not in other stages of the Artemia life cycle (Figure 1A). AMPK is a central component of a protein kinase cascade that plays a pivotal role in the regulation of the intracellular energy status. It has been estab-

A
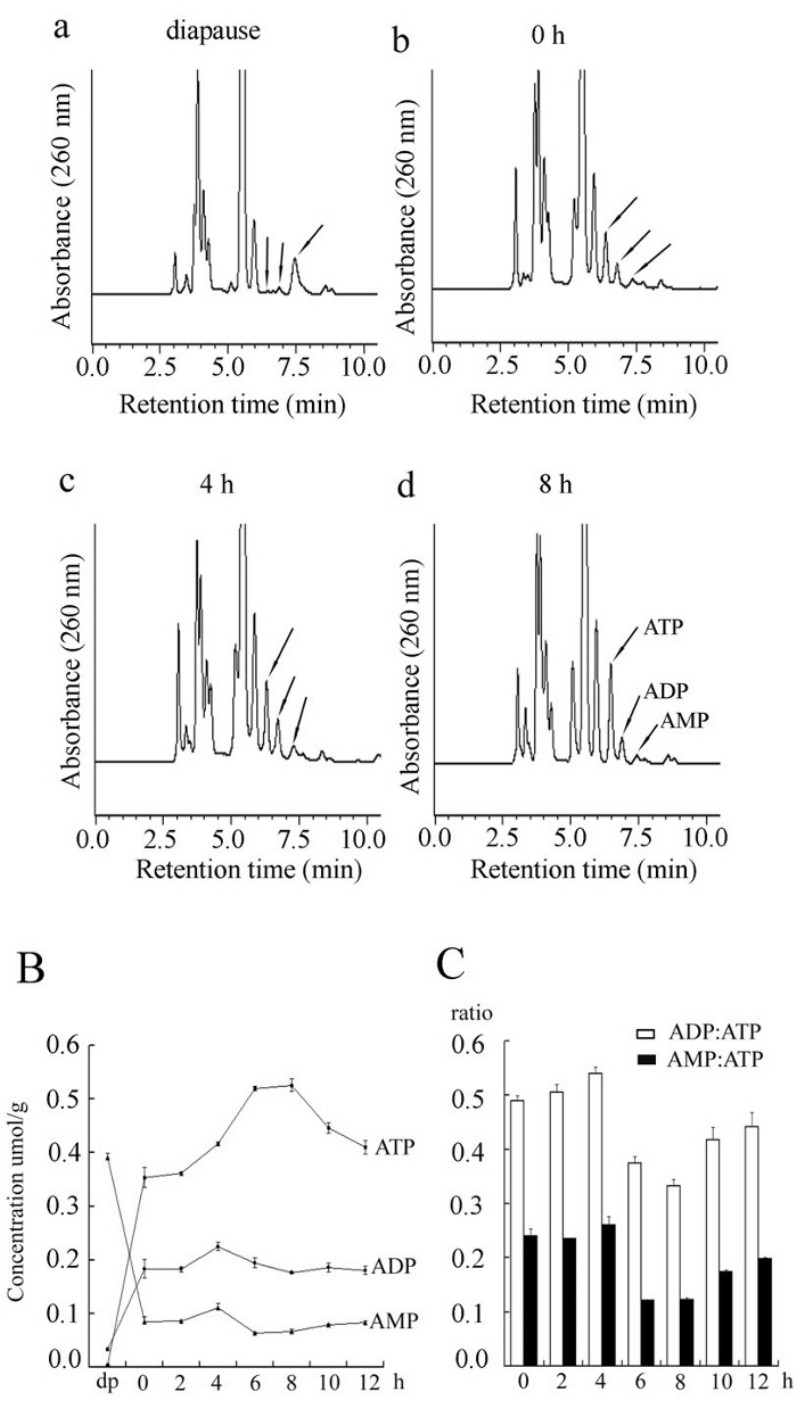

Figure 4

Cellular ATP, ADP, and AMP were determined by HPLC analysis. A. HPLC chromatogram of ATP, ADP, and AMP from Artemia diapause embryos (a) and embryos at three representative developing stages $(0,4$, and $8 \mathrm{~h}, \mathrm{~b}-\mathrm{d})$. Arrows indicate peaks of ATP, ADP, and AMP. B. Concentrations of cellular ATP, ADP, and AMP in embryos at different developmental stages were determined by an external standard method. dp, diapause embryos. C. Changes of ADP:ATP (white columns) and AMP:ATP (black columns) ratios in developing embryos.

lished that activation of AMPK is associated with increased expression of key metabolic proteins, such as hexokinase II, GLUT-4, and mitochondrial enzymes $[23,26]$. Thus, Artemia AMPK may be a potential candidate for an energy control function during the post-dia- 
pause developmental stages. However, the fact that Artemia AMPK activation was not detected before the 4th hour of development indicates that AMPK functions may be development-related.

As a downstream target of LKB1, AMPK was shown to be indispensable in embryogenesis. It has been found that AMPK $\alpha$-null mutants of Drosophila die before adulthood and are unable to reach the larval stage when both zygotic and maternal AMPK contributions are eliminated [27]. In C. elegans, orthologues of LKB1 (par-4) and AMPK (aak1, aak2) cooperate to regulate germline proliferation with somatic development during dauer formation [28]. Thus, Artemia AMPK may not only function as an energy regulator, but also plays other roles during post-diapause developmental stages. To further explore the role of AMPK in this process, we also conducted a whole-mount immunohistochemistry experiment in developing cysts. Interestingly, our results show that activated AMPK is predominantly restricted in the ectoderm region of embryos and the position of the activation loop changes as development proceeds (Figure 2). Thus, AMPK is probably involved in the embryonic development process, such as differentiation of certain cells and morphologic changes.

Studies have shown that AMPK may regulate gene expression through direct interaction with the nucleus $[15,16]$. The observation that Artemia AMPK localizes to the nucleus when activated (Figure 3) suggests that Artemia AMPK interacts with transcriptional regulators or DNA directly to control gene expression. This finding, combined with the results that activated Artemia AMPK exists in certain cells and then its location changes during development (Figure 2), led to the hypothesis that AMPK may participate in regulating the expression of genes that function in development of certain organs or tissues.

Thus far, two proteins (p26 and p8) have been established as nuclear binders in Artemia [1,29]. p8 is the first diapauses-related transcription factor to be identified in crustaceans, while p26, making up $10-15 \%$ of the total nonyolk protein of Artemia diapause embryos, serves primarily as a cell stress chaperone [29,30]. Interestingly, nuclear-cytoplasmic translocations of p26 are pHdependent. Additionally, previous work by Utterback and Hand [11] has shown that alteration of pHi influences yolk platelet degradation during post-diapause development in Artemia embryos. Thus, we also examined the $\mathrm{pH}-$ dependence of nuclear-cytoplasmic translocations of activated AMPK by manipulating buffer $\mathrm{pH}$ in vitro (Figure $3 \mathrm{~A})$. The results showed that the location of activated AMPK in the nucleus was not affected by pHi.

It is known that AMPK is activated by conditions leading to an increase in the intracellular AMP:ATP ratio [16].
However, our studies have shown that Artemia AMPK is not activated in diapause embryos in which the AMP concentration is rather high (Figure $4 \mathrm{~B}$ ), but is activated in developing cysts from the 4 th hour of post-diapause developmental stages in which the intracellular AMP:ATP ratio is low (Figure 4C). This result seems uncoupled to traditional mechanisms of AMPK activation by an elevated AMP:ATP ratio $[16,23]$. During the post-diapause developmental stages, however, it was notable that AMPK was only activated in certain cells of developing cysts, as revealed by the whole-mount immunohistochemistry results (Figure 2 ). There are two possible reasons for the activation of AMPK in this process: 1) although the AMP:ATP ratio is low in whole embryos, in the cells in which AMPK is activated, the AMP:ATP ratio is high; if so, AMPK activation is consistent with the classic regulatary mechanisms by the AMP:ATP ratio, and 2) although the AMP:ATP ratio is a major signal for AMPK activation, Artemia AMPK could also be regulated by other pathways which are independent of the cellular adenosine nucleotide level. This type of AMPK activation regulation by other cell signals has been reported in other organisms. In human cells, AMPK activity is regulated by two upstream kinases, LKB1 and CaMKK $[17,18]$. Regulation of the AMPK activity by CaMMK is not affected by the AMP:ATP ratio.

As is known, classic AMPK activation stimulates ATP-producing, catabolic pathways and inhibits ATP-consuming anabolic pathways [23]. Thus, it could be hypothesized that AMPK activation in the whole embryo during the post-diapause development process would inhibit ATPconsuming processes, such as protein synthesis and cell growth. However, it is notable that AMPK was only activated in certain cells (Figure 2). This fact indicates that even if Artemia AMPK is activated to switch off the ATPconsuming pathways in some cells, it would probably not inhibit the anabolic activity of the whole embryo. Furthermore, the fact that AMPK enters nuclei suggests that AMPK may play more roles in regulating the expression of the development-related genes rather than directly controlling cell energy. Considering the inhibition of anabolic metabolism by AMPK, it can also be hypothesized that the stimulation of AMPK contributes to metabolic depression and the inhibition of proliferation in the diapause embryos in which the AMP level is high and the metabolic activity is undetectable. However, AMPK was not detectably activated in the diapause state (Figure 1A). One possible reason is that AMPK is not the key factor that controls or maintains diapause. Thus, in the diapause state, as with other key metabolic enzymes, such as S6 kinase and hexokinase[25,31], AMPK loses its activity in spite of high AMP concentrations.

\section{Conclusion}

In summary, our study focused on AMPK activation during the Artemia life cycle. The spatial-temporal activation 
pattern of AMPK, as well as its subcellular location, helped to further investigate its functions in Artemia post-diapause developmental stages. Our findings may thus provide insight into the regulation of energy and gene expression during Artemia post-diapause development, and reveal further aspects of AMPK function.

\section{Methods \\ Culturing of Brine Shrimps and Embryos for Sampling}

Post-diapause embryos of Artemia franciscana, gifted by Freshwater Fisheries Institute of Zhejiang (China), were incubated in ice-cold artificial sea water (3\%) under continuous light at $25^{\circ} \mathrm{C}$ following hydratation at $4{ }^{\circ} \mathrm{C}$ for 5 hours. Sampling was done at 0, 2, 4, 6, 8, 10, and $12 \mathrm{~h}$, and at the free-swimming nauplius stage. Artemia were kept at room temperature $\left(25-27^{\circ} \mathrm{C}\right)$ and fed once a day with Chlorella powder. Encysted embryos were gathered and stored dry at $25^{\circ} \mathrm{C}$ as a sample set of diapause embryos and used within 2 weeks. All samples were used immediately or snap-frozen in liquid nitrogen and stored at $-80^{\circ} \mathrm{C}$ until use.

\section{Western Blotting on Whole Protein Extracts}

Proteins were extracted from each sample using the TRIZOL reagent (Invitrogen, Carlsbad, CA, USA) according to the manufacturer's instructions, and were quantified using the Bradford method [32]. Fifty $\mu \mathrm{g}$ of protein of each sample were separated on $10 \%$ SDS-polyacrylamide gels (SDS-PAGE) and transferred to PVDF membranes (Millipore, Bedford, MA, USA). The membranes were incubated with phospho-AMPK (Thr172) (1:1000; Cell Signaling Technology, Beverly, MA, USA) and $\alpha$-tubulin (1:1000; Beyotime, Shanghai, China) antibodies overnight at $4{ }^{\circ} \mathrm{C}$, and the detection was performed using the BM Chemiluminescence Western Blotting Kit (Roche, Mannheim, Baden-Wurttenberg, Germany).

\section{Expression of GST-SAMS}

For the expression of GST-SAMS, a recombinant plasmid was constructed. In brief, two oligos (5'GATCCCATATGCGCAGCGCGATGAGCGGCCTGCATCTGGTGAAACGCCGCTGAC-3' and 5'TCGAGTCAGCGGCGTTTCACCAGATGCAGGCCGCTCATCGCGCTGCGCATATGG-3', $10 \mu \mathrm{M}$ each) were mixed together in TN buffer (10 mM Tris [pH 8.0] and $10 \mathrm{mM}$ $\mathrm{NaCl}$ ), heated at $70^{\circ} \mathrm{C}$ for $5 \mathrm{~min}$, and then cooled down slowly to room temperature. The annealed product was then subcloned in pGEX4T1 (Amersham Biosciences) at BamHI and XhoI sites. The nucleotide sequence of the recombinant plasmid was confirmed by DNA sequencing. The GST-SAMS or GST was expressed in BL21(DE3)pLysS of $E$. coli strain using IPTG induction $(0.1 \mathrm{mM})$ at $20^{\circ} \mathrm{C}$ overnight. GST-SAMS or GST from $5 \mathrm{ml}$ of cell culture was bound to $50 \mu \mathrm{l}$ of glutathione beads using Glutathione Sepharose $^{\mathrm{TM}}$ 4B (GE Healthcare, Piscataway, NJ).

\section{In vitro kinase assay}

Embryos were homogenized in a homogenization buffer (20 mM Tris [pH 7.5], $150 \mathrm{mM} \mathrm{NaCl}, 1 \mathrm{mM}$ EDTA, $1 \mathrm{mM}$ EGTA, $250 \mathrm{mM}$ mannitol, $50 \mathrm{mM}$ sodium fluoride, $1 \%$ Triton X-100, $2.5 \mathrm{mM}$ sodium pyrophosphate, $1 \mathrm{mM}$ bglycerophosphate, $1 \mathrm{mM} \mathrm{Na3Vo4}$, and $1 \mathrm{ug} / \mathrm{ml}$ leupeptin). The homogenate was centrifuged and the supernatant was subjected to polyethylene glycol (PEG) precipitation.

AMPK activity was measured using the GST-SAMS phosphorylation assay. The kinase assay was performed at $30^{\circ} \mathrm{C}$ for $20 \mathrm{~min}$ with $100 \mu \mathrm{g}$ of PEG extracts in assay buffer containing $40 \mathrm{mM}$ HEPES, $0.2 \mathrm{mM} \mathrm{AMP,} 80 \mathrm{mM}$ $\mathrm{NaCl}, 8 \%$ glycerol, $0.8 \mathrm{mM}$ DTT, $5 \mathrm{mM} \mathrm{MgCl}_{2}$, and 0.2 mM ATP with 5 ul of GST-SAMS or GST binding beads. After reaction, the beads were quickly centrifuged and washed with TBS buffer. Then, the beads were boiled in $2 \times$ protein loading buffer for $5 \mathrm{~min}$ and centrifuged. The supernatants were separated by $15 \%$ SDS-PAGE, and phosphorylated proteins were detected by Western blotting using Biotinylated Phos-tag ${ }^{\mathrm{TM}}$.

\section{Immunohistochemistry on whole mount embryos}

For whole mount immunohistochemical studies, cysts were decapsulated using antiformin [22]. Decapsulated embryos were fixed in 4\% paraformaldehyde, and permeated by $0.1 \%$ Triton X-100. Phospho-AMPK (Thr172) antibody (1:100) was used as the primary antibody. The secondary antibody was AP-conjugated goat anti-rabbit IgG secondary antibody (1:350; Promega, Madison, WI, USA). The staining was performed using the NBT/BCIP solution in the dark.

\section{Nuclear translocation of AMPK}

Cell fractionation was performed as described [22]. Briefly, 4-h embryos were homogenized using Dounce homogenizers in buffer $\mathrm{K}$ ( $\mathrm{pH} 6.0$ or 8.0 ). Homogenates were centrifuged at $1630 \mathrm{~g}$ for $5 \mathrm{~min}$ at $2^{\circ} \mathrm{C}$ to obtain supernatant and pellet fractions. Pellets (nuclei, yolk platelets, and shell fragments) were washed once with 200 times volume of buffer $\mathrm{K}$ and restored to initial volumes. After being heated in $2 \times$ sample buffer at $100^{\circ} \mathrm{C}$ for $5 \mathrm{~min}$, insoluble shell fragments were removed by centrifugation at $1630 \mathrm{~g}$ for $5 \mathrm{~min}$ [33]. Supernatants were electrophoresed in 10\% SDS-PAGE, and proteins were detected by Coomassie blue-G staining. Proteins from SDS-PAGE were also transferred to PVDF membranes and incubated with Phospho-AMPK (Thr172) antibody as described above.

\section{Immunofluorescent Staining of Nuclei}

Nuclei of 6-h embryos were prepared by centrifugation on Percoll gradients, fixed in $4 \%(\mathrm{w} / \mathrm{v})$ paraformaldehyde, and processed as described [3]. Phospho-AMPK (Thr172) 
antibody (1:100) was used as the primary antibody. The secondary antibody was FITC-conjugated goat anti-rabbit IgG (1:100; Sigma, St. Louis, MO, USA). After a 30-min incubation with secondary antibody, the nuclei were rinsed with PBS, and incubated with DAPI (Beyotime, Shanghai, China). Slides were examined with either a Nikon ECLIPSE TE200-S microscope with an argon/krypton laser or a Zeiss LSM 510 inverted laser scanning confocal microscope.

\section{Determination of ATP, ADP, and AMP Concentrations}

Diapause embryos and post-diapause embryos that developed for $0,2,4,6,8,10$, and $12 \mathrm{~h}$ were collected and rinsed by sterile water. Each sample contained at least 20 $\mathrm{mg}$ of embryos. Excessive water was removed before adenylate (ATP, ADP, and AMP) extraction with perchloric acid [34]. The HPLC conditions were as follows: a SHIMADZU CLC-C8 250*4.60 mm column was equipped with a SHIMADZU LC-10AT pump system. Mobile phase A consisted of $0.18 \mathrm{M} \mathrm{K}_{2} \mathrm{HPO}_{4}$ and $0.1 \mathrm{M} \mathrm{KH}_{2} \mathrm{PO}_{4}$ dissolved in deionized water, while mobile phase $\mathrm{B}$ consisted of $65 \%$ acetonitrile. The flow rate of the mobile phase was $1 \mathrm{ml} / \mathrm{min}$, while the injection volume was $10 \mu \mathrm{l}$. Peaks were detected and analyzed at $260 \mathrm{~nm}$, and the column temperature was $30^{\circ} \mathrm{C}$. The elution program was as follows: 0 min $100 \%$ A, 0\% B; 10 min $100 \%$ A, 0\% B; 18 min $0 \% \mathrm{~A}, 100 \% \mathrm{~B} ; 23$ min 0\% A, 100\% B; 25 min 100\% A, $0 \% \mathrm{~B}$; and 35 min stop. ATP, ADP, and AMP were identified by comparison with retention time of standards (Sangon, Shanghai, China) while their concentrations were determined using the external standard method. Data was expressed as the means \pm s.e.m. of three replicate determinations.

\section{Authors' contributions}

XJZ designed and performed the experiments, and wrote the paper. JQD participated in the Western blotting analysis and the whole mount immunohistochemistry. XT participated in the determination of adenylate concentrations. YZ participated in the kinase activity assay. WJY supervised and participated in the design of the study. All authors read and approved the final manuscript.

\section{Additional material}

\section{Additional file 1}

Phosphorylated AMPK is found in different layers of nuclei (a-o) from 4$h$ incubated embryos by immunofluorescent staining of nuclei and confocal microscopy. The data provide confocal microscopy images of sections of a single nucleus double-stained with DAPI and FITC-conjugated goat anti-rabbit IgG after incubation with phospho-AMPK (Thr172) antibody. Click here for file

[http://www.biomedcentral.com/content/supplementary/1471213X-9-21-S1.jpeg]

\section{Additional file 2}

Concentrations of cellular ATP, ADP, and AMP in embryos at different developmental stages ( $0-12 \mathrm{~h} ; \mathrm{m}$ per $1 \mathrm{~g}$ wet weight embryos). The data provided represent the statistical analysis of cellular adenylates concentration in various embryos.

Click here for file

[http://www.biomedcentral.com/content/supplementary/1471213X-9-21-S2.doc]

\section{Acknowledgements}

We thank Dr. Hanmin Chen of Zhejiang University for his assistance in conducting the confocal microscopy observation. We also thank Chris Wood of The College of Life Sciences of Zhejiang University for critical reading of this manuscript.

This work was supported by the National Natural Sciences Foundation of China (407302I 2 and 40876069), and the '863' program of China (2007AA09Z426).

\section{References}

I. Liang P, Amons R, Clegg JS, MacRae TH: Molecular characterization of a small heat shock/alpha-crystallin protein in encysted Artemia embryos. J Biol Chem 1997, 272:1905I-8.

2. Liang P, MacRae TH: The synthesis of a small heat shock/alphacrystallin protein in Artemia and its relationship to stress tolerance during development. Dev Biol 1999, 207:445-56.

3. Willsie JK, Clegg JS: Nuclear p26, a small heat shock/alpha-crystallin protein, and its relationship to stress resistance in Artemia franciscana embryos. J Exp Biol 200I, 204:2339-50.

4. Clegg JS, Jackson SA, Liang P, MacRae TH: Nuclear-cytoplasmic translocations of protein p26 during aerobic-anoxic transitions in embryos of Artemia franciscana. Exp Cell Res 1995, 219:1-7.

5. Warner AH, Brunet RT, MacRae TH, Clegg JS: Artemin is an RNAbinding protein with high thermal stability and potential RNA chaperone activity. Arch Biochem Biophys 2004, 424: $189-200$.

6. Kwast $\mathrm{KE}, \mathrm{Hand} \mathrm{SC}$ : Oxygen and $\mathbf{p H}$ regulation of protein synthesis in mitochondria from Artemia franciscana embryos. Biochem J 1996, 3 I3(Pt I):207-I3.

7. Hand SC: Oxygen, $\mathbf{p H i}$ and arrest of biosynthesis in brine shrimp embryos. Acta Physiol Scand 1997, 16 1:543-51.

8. Nakanishi YH, Iwasaki T, Okigaki T, Kato H: Cytological studies of Artemia salina. I. Embryonic development without cell multiplication after the blastula stage in encysted dry eggs. Annot Zool Jpn 1962, 35:223-228.

9. Nakanishi YH, Okigaki T, Kato H, Iwasaki T: Cytological studies of Artemia salina. II. Deoxyribonucleic acid (DNA) content and the chromosomes in encysted dry eggs and nauplii. Proc Jpn Acad 1963, 39:306-309.

10. Wang W, Meng B, Chen W, Ge X, Liu S, Yu J: A proteomic study on postdiapaused embryonic development of brine shrimp (Artemia franciscana). Proteomics 2007, 7:3580-91.

II. Utterback PJ, Hand SC: Yolk platelet degradation in preemergence Artemia embryos: response to protons in vivo and in vitro. Am J Physiol 1987, 252:R774-8I.

12. Santiago J, Sturgill TW: Identification of the S6 kinase activity stimulated in quiescent brine shrimp embryos upon entry to preemergence development as p70 ribosomal protein S6 kinase: isolation of Artemia franciscana p70S6k cDNA. Biochem Cell Biol 200I, 79: 141-52.

13. Clegg JS, Golub AL: Protein synthesis in Artemia salina embryos. II. Resumption of RNA and protein synthesis upon cessation of dormancy in the encysted gastrula. Dev Biol 1969, 19:178-200. 
14. Clegg JS: The Control of Emergence and Metabolism by External Osmotic Pressure and the Role of Free Glycerol in Developing Cysts of Artemia Salina. J Exp Biol 1964, 41:879-92.

15. Hardie DG: New roles for the LKBI-->AMPK pathway. Curr Opin Cell Biol 2005, I7:167-73.

16. Hardie DG: The AMP-activated protein kinase pathway - new players upstream and downstream. / Cell Sci 2004, I I 7:5479-87.

17. Woods A, Johnstone SR, Dickerson K, Leiper FC, Fryer LG, Neumann D, Schlattner U, Wallimann T, Carlson M, Carling D: LKB I is the upstream kinase in the AMP-activated protein kinase cascade. Curr Biol 2003, 13:2004-8.

18. Woods A, Dickerson K, Heath R, Hong SP, Momcilovic M, Johnstone SR, Carlson M, Carling D: Ca2+/calmodulin-dependent protein kinase kinase-beta acts upstream of AMP-activated protein kinase in mammalian cells. Cell Metab 2005, 2:2I-33.

19. Ramamurthy S, Ronnett GV: Developing a Head for Energy Sensing: AMP-activated Protein Kinase as a Multifunctional Metabolic Sensor in the Brain. J Physiol 2006, 574:85-93.

20. Zhu XJ, Feng CZ, Dai ZM, Zhang RC, Yang WJ: AMPK alpha subunit gene characterization in Artemia and expression during development and in response to stress. Stress 2007, 10:53-63.

21. Kishimoto A, Ogura T, Esumi $\mathrm{H}$ : A pull-down assay for 5' AMP. activated protein kinase activity using the GST-fused protein. Mol Biotechnol 2006, 32:17-2I.

22. Clegg JS, Jackson SA, Warner AH: Extensive intracellular translocations of a major protein accompany anoxia in embryos of Artemia franciscana. Exp Cell Res 1994, 2 I 2:77-83.

23. Hardie DG, Salt IP, Hawley SA, Davies SP: AMP-activated protein kinase: an ultrasensitive system for monitoring cellular energy charge. Biochem J 1999, 338(Pt 3):717-22.

24. Nambu Z, Tanaka S, Nambu F: Gene expression of trehalase during post-dormant development of the brine shrimp, Artemia: comparison of the two species. J Uoeh 1997, 1 9:255-64.

25. Malarkey K, Coker KJ, Sturgill TW: Ribosomal S6 kinase is activated as an early event in preemergence development of encysted embryos of Artemia salina. Eur J Biochem 1998, 25 I:269-74.

26. McGee SL, Howlett KF, Starkie RL, Cameron-Smith D, Kemp BE, Hargreaves M: Exercise increases nuclear AMPK alpha2 in human skeletal muscle. Diabetes 2003, 52:926-8.

27. Forcet C, Billaud M: Dialogue between LKBI and AMPK: a hot topic at the cellular pole. Sci STKE 2007, 2007:e5I.

28. Narbonne P, Roy R: Inhibition of germline proliferation during C. elegans dauer development requires PTEN, LKBI and AMPK signalling. Development 2006, 133:6I I-9.

29. Qiu Z, MacRae TH: Developmentally regulated synthesis of p8, a stress-associated transcription cofactor, in diapause-destined embryos of Artemia franciscana. Cell Stress Chaperones 2007, I 2:255-64.

30. Liang P, Amons R, Macrae TH, Clegg JS: Purification, structure and in vitro molecular-chaperone activity of Artemia p26, a small heat-shock/alpha-crystallin protein. Eur J Biochem 1997, 243:225-32.

3I. Rees BB, Ropson IJ, Hand SC: Kinetic properties of hexokinase under near-physiological conditions. Relation to metabolic arrest in Artemia embryos during anoxia. J Biol Chem 1989, 264: 15410-7.

32. Bradford MM: A rapid and sensitive method for the quantitation of microgram quantities of protein utilizing the principle of protein-dye binding. Anal Biochem 1976, 72:248-54

33. Laemmli UK: Cleavage of structural proteins during the assembly of the head of bacteriophage T4. Nature 1970, 227:680-685.

34. Liu H, Jiang Y, Luo Y, Jiang W: A Simple and Rapid Determination of ATP, ADP and AMP Concentrations in Pericarp Tissue of Litchi Fruit by High Performance Liquid Chromatography. Food Technol Biotechnol 2006, 44:531-534.

\footnotetext{
Publish with Biomed Central and every scientist can read your work free of charge

"BioMed Central will be the most significant development for disseminating the results of biomedical research in our lifetime. "

Sir Paul Nurse, Cancer Research UK

Your research papers will be:

- available free of charge to the entire biomedical community

- peer reviewed and published immediately upon acceptance

- cited in PubMed and archived on PubMed Central

- yours - you keep the copyright

Submit your manuscript here:

http://www.biomedcentral.com/info/publishing_adv.asp

BioMedcentral
} 